Furthermore the buildup of secondary perturbations may be speeded up by a three-dimensional process, such as stretching of the vortex lines in the direction of the $z$ axis.

It would be interesting to stimulate the secondary perturbations by some artificial means (say by sound waves), and to observe the response of the oscillating boundary layer. In particular the phase of the secondary perturbation may lock with that of the stimulus.

${ }^{1}$ P. S. Klebanoff and K. D. Tidstrom, "The evolution of amplified waves leading to transition in a boundary layer with zero-pressure gradient." N.A.S.A. Tech. Note D-195 (1959).

2 R. Betchov, J. Ship Research (in print).

${ }^{3}$ R. E. Esch, J. Fluid Mech. 3, 289 (1957).

${ }^{4}$ P. S. Klebanoff (private communication).

\section{Use of Fine Unheated Wires in Shock Tubes}

\author{
Walter H. Christiansen \\ Guggenheim Aeronautical Laboratory, \\ California Institute of Technology, \\ Pasadena, California \\ (Received August 15, 1960)
}

$\mathrm{S}^{\mathrm{O}}$ OME preliminary measurements of heat transfer to fine unheated wires have been made in the shock tube. These measurements show that the response of the wire is nearly exponential with time.

The wire is physically similar to an ordinary hot-wire anemometer, but conceptually its application is quite different. The gauge uses only a small excitation current to produce a voltage signal across the wire and it will practically maintain the temperature of its surroundings before the initiation of the hot flow. After the shock wave passes over the wire, the wire begins to heat up in this flow. The heating continues until the wire reaches an equilibrium temperature or until the hot flow ceases. The wire is assumed to be a perfect calorimeter, that is, all the heat convected to the wire is stored in the wire itself. Calorimetric methods of heat transfer have been used before in shoek tubes.1,2 The resulting change of wire temperature produces a resistance change of the wire which is conveniently read as a voltage on an oscilloscope.

The response time of the instrument is limited by two characteristic times: (1) The time required to establish flow over the wire is of order $10 \mathrm{~d} / U$. For the larger wire sizes this time is approximately 1 usec. (2) The characteristic time it takes to heat the wire uniformly throughout its volume is given by $t \sim \rho c d^{2} / k$. For a $\frac{1}{2}$-mil platinum wire, this time is $1.6 \mu \mathrm{sec}$. Hence under most conditions in the shock tube, even those that involve small testing times $0(10 \mu \mathrm{sec})$, the wire can be considered in steady flow and uniform in temperature.

A temperature rise of the wire is accompanied by a resistance variation of the wire. If this variation is moder-

ate $\left(\Delta T^{\prime}\right.$ of the order of $\left.200^{\circ} \mathrm{C}\right)$, the relation between resistance and temperature is nearly linear. The variation in voltage across the wire is then

$$
\Delta E=I R_{i} \alpha_{i}\left(T_{w}-T_{i}\right)
$$

for constant current operation. The subscript $i$ denotes some initial temperature where the properties of the wire are known. Usually this reference is room temperature.

An ordinary heat balance of an infinitely long wire gives the simple equation

$$
\frac{d}{d t}\left[\frac{1}{4} \pi d^{2} l \rho c\left(T_{u r}-T_{i}\right)\right]=Q_{\text {foreed convection }}
$$

The Joule heating due to the constant current $I$ is not included in the equation because it is negligible compared to the heat rates that are experienced in the shock tube. Hot-wire results ${ }^{3}$ show that the Nusselt number is nearly constant for fixed flow conditions and all heat transfer rates (i.e., varying temperature loadings). With the definition of the Nusselt number, Eq. 2 can be integrated to give

$$
T_{z w}=T_{r}-\left(T_{r}-T_{s}\right) e^{-4 \text { Nukl/dsc }},
$$

where $T_{r}$ is the wire recovery temperature. The quantity $d^{2} \rho c / 4 \mathrm{Nu} k=\tau$ is referred to as the time constant of the wire. Time constant values range from 0.1 to $10 \mathrm{msec}$ in the shock tube, and usually these times are longer than the uniform flow times encountered in the shoek tube.

After the passage of the initial shock wave the wire response should follow Eq. 3. If the flow time is short compared to $\tau$, the heat transfer is nearly constant and the response is linear. If the flow time is long compared to the time constant the response is exponential. Figure 1 shows both conditions. These figures also show the termination of the hot flow. This is denoted by the first discontinuity after the arrival of the shock.

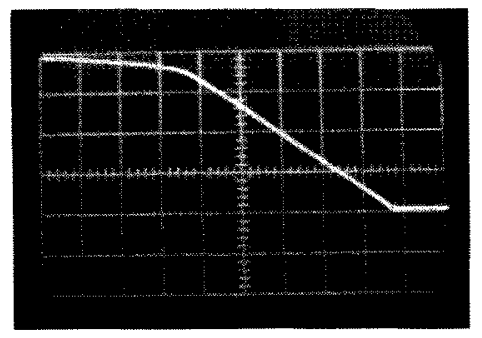

(a)

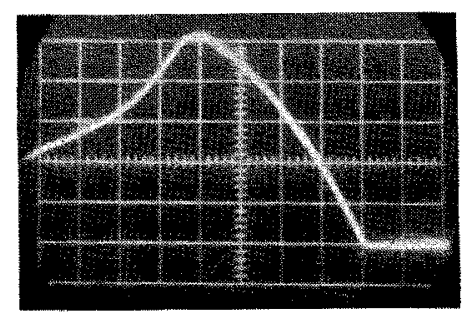

(b)
FIG. 1. Typical gauge responses. (a) $t / \tau \ll 1$. Sweep: 100 $\mu \mathrm{sec} / \mathrm{cm}$. Experimental flow duration: $500 \mu \mathrm{sec} ; \tau=$ $4370 \mu \mathrm{sec} ; t / \tau=$ 0.114 . (b) $t / \tau \simeq 1$. Sweep: $100 \mu \mathrm{sec} /$ cm. Experimental flow duration: 380 $\mu$ sec; $\tau=405$ $\mu \mathrm{sec} ; t / \tau=0.94$. 
The forced convection heat transfer rate per unit area is given by

$$
q=\frac{d \rho c}{4 \alpha_{i}} \frac{1}{I R_{i}} \frac{d E}{D t}
$$

Hence the heat transfer per unit area is directly proportional to the time rate of change of voltage across the wire. The heat transfer to the wire decreases exponentially with time. The wire never actually reaches equilibrium because of the short duration of flow time and hence the heating stops after the hot flow ceases. The heat transfer rate that is most significant occurs at $t=0$ (i.e., just after the arrival of the shock wave). There are two reasons for this: (1) The temperature of the wire is known at this time $\left(T_{w}=T_{i}\right)$ and does not need to be calculated. (2) There is no heat lost to the wire supports at $t=0$ and therefore no end loss corrections are necessary.

Preliminary heat transfer results of several runs are shown in Fig. 2. For the pressures and Mach numbers

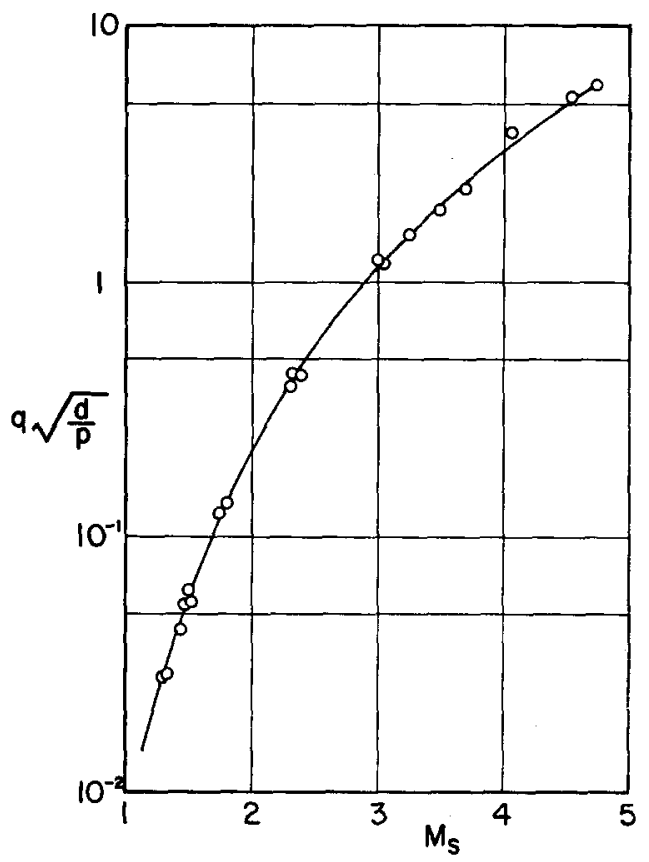

FIG. 2. Heat transfer rate versus shock Mach number. Nominal diameter of wires used in the experiment: 0.0025 , $0.00125,0.0005 \mathrm{~cm}$. Initial pressures of the shock tube: 5,50 , $500 \mathrm{~mm} \mathrm{Hg}$. Dimensions of heat transfer rate parameter: $\left(\mathrm{cal} / \mathrm{cm}^{2} \mathrm{sec}\right)(\mathrm{cm} / \mathrm{mm} \mathrm{Hg})^{\frac{1}{2}}$.

shown the heat transfer rate is proportional to $(p / d)^{3}$ as one would expect from hot-wire results. The results are quite consistent and repeatable. The wire should prove to be a valuable flow instrument in many shortduration flow problems with a gas of high stagnation enthalpy. Further investigations of this are being carried out. A more detailed report including calibration technique, construction of the wire, end loss corrections, and operating technique may be found in reference 4.
This work was carried out under the sponsorship and with financial assistance of the office, Chief of Ordnance, and Office of Ordnance Research, U. S. Army.

1 P. H. Rose, "Development of the calorimeter heat transfer gage for use in the shock tubes," Avco Research Rept. 17, February 1958.

${ }_{2}^{2}$ P. H. Rose, and W. I. Stark, J. Aeronaut. Sci., 25, 86 (1958).

${ }^{3}$ J. Laufer, and R. McClellan, J. Fluid Mech. 1, 276 (1956)

4 W. H. Christiansen, Galcit Hypersonic Research Project, Memo. No. 55, June 1, 1960.

\section{Electrical Discharges in Hypersonic Flows}

\author{
Gary Marlotte and A. Demetriades \\ Guggenheim Aeronautical Laboratory, \\ California Institute of Technology, \\ Pasadena, California
}

(Received July 6, 1960)

$\mathrm{N}$ order to study the interaction between an electrical current and a high-speed flow, experiments are being conducted with low-current electrical discharges in the $5 \times 5$-in. test section of the GALCIT hypersonic tunnel, in which continuous uniform flows are produced at $M=5.8$ and total temperatures $=150^{\circ} \mathrm{C}$.

One of the very first problems investigated was the dielectric breakdown of air between two electrodes immersed in the hypersonic stream. Studies were made with several pairs of copper and tungsten electrodes, with their electric field aligned either parallel with or transverse to the stream. Figure 1 shows breakdown voltages obtained with uncooled copper electrodes designed in the "Rogowski" fashion, for the case where the electric field vector was transverse to the airstream. Contrary to initial expectation the breakdown voltages are seen to lie below those obtained statically (without flow) with the same geometry. A more important observation is that the Paschen similarity criterion, which states that the breakdown voltage is solely a function of the product of the gas density and the interelectrode distance, is not borne out here.

An apparent explanation of the data lies in the choice of the density in the product $\rho d$. High-speed flows can support large density differences across shock waves and boundary layers continuously, and therefore the assumption of uniform density inherent in the static breakdown analysis is no longer true. In the present case one has to take into account the local Mach number and laminar boundary-layer thickness over the electrode; in other words, the Paschen similarity should now be extended to include aerodynamic similarity.

A simple analysis was carried out, resulting in the computation of breakdown voltages for uniform electric field but nonuniform density field between the electrodes. 